observed path and on wind profiles suggest that chunks weighing tens of kilograms probably landed close to the village of Travniki, and one weighing around 1 kilogram may have hit northwest of the village of Shchapino, Borovicka says. Thousands of smaller pieces may be hiding in a 25 -kilometre-long swathe south of the final point of the trajectory.
The largest single fragment, a piece of rock that could weigh up to half a tonne, may have landed smack in Lake Chebarkul. A 6-metrewide hole found in the lake's frozen surface the morning after the impact "almost certainly" marks the spot where the whopper came down, says Borovicka.

Russian military divers are busy searching the shallow lake. But Spahr, who discussed the impact with other scientists at a meeting of the United Nations Committee on the Peaceful Uses of Outer Space in Vienna last month, isn't so sure. "From what we've seen on pictures, the 'crater' just doesn't look right," he says. "It looks more like a hole someone has cut in the lake with an axe." -

\title{
Metal oxide chips show promise
}

\section{Materials that flip from insulator to conductor could make energy-efficient transistors.}

\section{BY EUGENIE SAMUEL REICH}

$\mathrm{T}$ he switches in most electronic circuits are made of silicon, one of the commonest elements. But their successors might contain materials that, for now, are lab-grown oddities: strongly correlated metal oxides.

The allure of these materials lies in the outer shells of electrons surrounding their metal atoms. The shells are incomplete, leaving the electrons free to participate in coordinated quantum-mechanical behaviour. In some materials, electrons pair up to produce superconductivity, or coordinate their spins to produce magnetism. Other materials can switch from being an insulator to a conductor.

Unlike transitions to superconductivity, which happen as temperatures approach absolute zero, the insulating-to-conducting transition typically happens as temperature increases, and sometimes occurs near room temperature. That has raised hopes that metal oxides could be used instead of silicon to make transistors. A spate of results is now making that look feasible. "People are interested in seeing if oxides can make it to applications," says Manuel Bibes, a physicist at the Joint Physics Unit in Palaiseau, France, which is run by the French National Research Centre and electronics company Thales.

Metal oxide transistors have the potential to consume less power than silicon switches, because the phase transition frees electrons from their localized state near each atom, without moving them through the bulk material. By contrast, silicon switches work by pulling electrons through the material to a channel where they conduct current (see 'Go with the flow').

In the past 5-10 years, researchers have succeeded in growing high-quality thin films of the metal oxides - overcoming one of the major barriers to applications. In July 2012, for example, a group in Japan reported ${ }^{1}$ that it had deposited a thin film of vanadium dioxide that underwent a phase transition in response to an

\section{GO WITH THE FLOW}

Metal oxide transistors have the potential to consume less power than silicon ones, because switching does not require the atoms to be relocated.

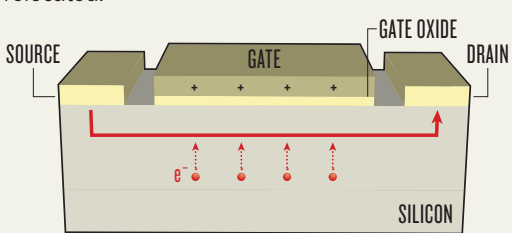

Silicon transistor

A positive charge at the gate draws electrons into a surface channel, allowing current to flow between the source and the drain.

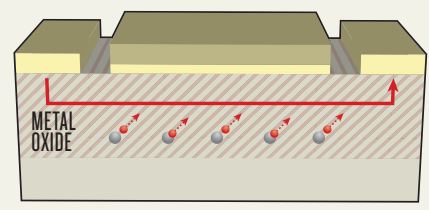

Metal oxide transistor

An applied electric field at the gate causes electrons on metal atoms to become conductive, letting current flow between source and drain.

applied electric field - proof that the material could be used as an electronic switch.

And last month, a group led by Shriram Ramanathan, a materials scientist at Harvard University in Cambridge, Massachusetts, addressed a fabrication challenge by growing a thin film of samarium nickelate on top of a substrate made of silicon and silicon dioxide 2 .

The nickelate was deposited at a relatively low temperature that did not disturb the underlying silicon layers, raising the possibility of manufacturing metal oxides on top of silicon wafers to form three-dimensional chips, says Andrew Millis, a solid-state theorist at Columbia University in New York. Not only would that allow computing power to be packed much more densely, says Millis, but it would also permit metal oxide switches to be built on top of existing circuit architectures.

Other groups are trying to understand the nature of the phase transition. In January, Ivan Schuller, a solid-state physicist at the University of California, San Diego, and his colleagues showed $^{3}$ that in vanadium oxide, the transition is in large part caused by micrometre-scale heating by the applied electric field.

Some point to Schuller's work as evidence that metal oxides will never make fast switches, because heating effects are usually quite slow. But Ramanathan says that his own measurements on vanadium oxide demonstrate that the phase transition is quite fast - less than a few nanoseconds - and that it should not hinder applications.

Some physicists are finding further examples of potentially useful materials. Bernhard Keimer at the Max Planck Institute for Solid State Research in Stuttgart, Germany, alternates thin layers of metal oxides to form composites that often turn out to have serendipitous properties. His group layered conducting lanthanum nickelate and insulating lanthanum aluminate and found ${ }^{4}$ that the composite underwent a transition between the two properties.

The highest phase-transition temperature for the composite was 150 kelvin above absolute zero - too low for practical applications. But the group is now trying to replicate the phenomenon in other materials that might have higher transition temperatures.

Sandip Tiwari, an applied physicist at Cornell University in Ithaca, New York, acknowledges that metal oxides are not yet close to competing with silicon. But given recent progress, he feels that researchers need to start trying to implement them in devices. That way, he says, all the properties needed for a good transistor will be developed in tandem. "If you just look at whatever property is your favourite, you won't get them all."

1. Nakano, M. et al. Nature 487, 459-462 (2012).

2. Jaramillo, R., Schoofs, F., Ha, S. D. \& Ramanathan,

S. J. Mater. Chem. C http://dx.doi.org/10.1039/ C3TC00844D (2013).

3. Zimmers, A. et al. Phys. Rev. Lett. 110, 056601 (2013).

4. Boris, A. V. et al. Science 332, 937-940 (2011). 